\title{
Bellman-Ford algorithm for solving shortest path problem of a network under picture fuzzy environment
}

\author{
Mani Parimala $^{1}$ (D) Said Broumi ${ }^{2} \cdot$ Karthikeyan Prakash $^{1} \cdot$ Selçuk Topal $^{3}$
}

Received: 14 October 2020 / Accepted: 5 June 2021 / Published online: 13 June 2021

(c) The Author(s) 2021

\begin{abstract}
An elongation of the novel intuitionistic fuzzy set is a picture fuzzy set theory. The demonstration of this has been used to deal with the abstinence criteria in a decision-making problem. The uncertainty in nature occurs sometimes in real-world problems and amidst them, the prominent one is the shortest path problem (SPP) solving. In the last few years, one of the best algorithms on the network for finding SPP is Bellman-Ford. Due to uncertainty in the decision-making process, it becomes difficult for decision-makers for communicating their point of view and judgment with certainty. We conceive of SPP in this contribution via Bellman's algorithm (BA) for a network with trapezoidal picture fuzzy numbers (TPFNs). We introduce a new algorithm to stand the shortest picture fuzzy path between each pair of nodes. A TPFN is considered for the length of all edges. A numerical example for the validation of the presented algorithm has also been proposed. There has also been relative research with existing techniques showing the benefits of the new algorithm.
\end{abstract}

Keywords Shortest path problem · Bellman-Ford algorithm $\cdot$ Picture fuzzy graph · Trapezoidal picture fuzzy number

\section{Introduction}

The short transmission distance of multifunction sensors in computer electronics and wireless communication triggers the development of low power and cost, multi-functional sensor nodes. Sensor nodes make possible environment sensing in conjunction with data processing. Like temperature, humidity, and volatile compound detection, with various instrumentations of sensors, let monitoring of different environments exist. The sensors are capable of handling

Mani Parimala

rishwanthpari@gmail.com

Said Broumi

broumisaid78@gmail.com

Karthikeyan Prakash

prakashk@bitsathy.ac.in

Selçuk Topal

s.topal@beu.edu.tr; selcuk.topal@yandex.com

1 Department of Mathematics, Bannari Amman Institute of Technology, Sathyamangalam 638401, India

2 Laboratory of Information Processing, Faculty of Science Ben M'Sik, University Hassan II, Casablanca, Morocco

3 Department of Mathematics, Arts and Sciences Faculty, Bitlis Eren University, Bitlis, Turkey networks with exchange data with external users and other sensor systems. Wireless networking, embedded systems, distributed processing, microelectromechanical systems, and wireless sensor applications are key features of this technology. Advances and innovations in sensor technology and the resulting large transformations have contributed greatly to wireless sensor networks. In the context of both business and our daily life, the sensors support and improve working performance. The sensor network device is far from the realtime situation, and a large amount of data can be obtained and processed. Sensory awareness is used by these sensors. The sensor's network and algorithm must have the ability to organize themselves.

Neighborhood nodes (vertices) are similar to each other, and nodes are used for continuous sensing. Unlike other sensors, multi-chip sensor networks are accustomed for low power consumption. The sensor network's each node provides the topological information. An interconnection network can be used for parallel computing. Shortest path algorithms are used for messages from any source to any destination. In most cases, BA is used for a network with wide-ranging secure nodes.

A set whose components have membership degrees called the fuzzy set (FS) [1] presented in 1965, which manages real-time issues where there is typically some kind of 
ambiguity in details. A 2-decade later, Atanassov in 1986 [2] broadened this definition to the intuitionistic fuzzy set (IFS), which is defined by the set's characteristic functions in terms of membership (MS) and non-membership (NMS) functions.

The notion of fuzzy numbers (FNs) has been introduced by Zadeh [3]. The concept of FNs by different circumstances was studied by Mizumoto and Tanaka [4], Dubois and Prade [5], and Nahmias [6], Mp et al. [7], among others. Burillo et al. [8] defined intuitionistic fuzzy numbers (IFNs) as an extension in line with the novel structure of fuzzy numbers. The arithmetic structures, and their properties were studied by Chen and Tan [9], Hong and Choi [10] and Shu-Xi [11]. The functioning laws and algorithms for intuitionist triangular FNs and the intuitionist fuzzy setting that explains faulttree analysis were suggested by Shu et al. [11]. To signify the membership function, Zhang and Liu [12] used the concept of triangular FN Functions, which put forward them to bring the concept of triangular IFN functions. Wang and Zhang [13] proposed the concepts of intuitionistic trapezoidal fuzzy number (ITFN) and interval-valued intuitionistic trapezoidal fuzzy number. Some aggregation operators on ITFN have been identified by Jian-qiang and Zhong [14]. PSO optimization of interval type-2 fuzzy controllers for FPGA applications was designed, simulated, and implemented by Yazmin Maldonado [15]. Purcaru et al. [16] developed an algorithm for path planning and the proposed algorithm has been used in a practical problem such as to calculate an ideal route for a robot that moves from an initial node to a destination node while eliminating all established environmental barriers.

Bellman [17], Dijkstra [18], Warshall [19], and Floyd [20] proposed some typical algorithms for solving SPPs. The solution for fuzzy shortest path problems (FSPP) can be found using the Bellman dynamic programming (BDP) method and a multiple objective linear programming problem was proposed by De and Bhincher [5] to consider the weights of the edges as uncertain or fuzzy for a network [21]. Many scientists have solved the SPP under environments like fuzzy and intuitionist fuzzy [22-24]. The Bellman algorithm definition has been used to solve SPP in a fuzzy network [25].

In different fields, Atanassov's IFS principle has been successfully applied. However, there are instances where a portion of abstinence linking two extreme conditions is often to be emphasized (Alcantud and Laruelle [26] for real reasons in terms of voting). It is normal to include an additional degree of freedom to the IFS concept to achieve this goal. A generalization of IFSs of this kind, called a picture fuzzy set (PFS), was introduced and studied by Cuong [27-29]. Considerably, PFS-related models are helpful when it is appropriate to integrate the view of professionals giving ambiguous responses in the manner: yes, abstain, no, and rejection. There has been some progress in the theory of PFS so far. Singh [30] tested the
PFS correlation coefficients and used them in the study of clustering. Son and Thong [31] presented a PF clusteringbased new hybrid forecast approach. Thong and Son [32] explored multivariable fuzzy forecasting using PF clustering and the PF rule interpolation technique. Akram and Zafer [33] contributed fascinatingly to readers. Akram et al. [34] presented and tested the Dijkstra algorithm using a TPFN of a picture fuzzy environmental network. Mani et al. [35] introduced the hesitancy fuzzy digraph and studied its operations with an example. This motivated us to work on this.

The Bellman dynamic programming (BDP), a mathematical formulation for unveiling the weighted aggregated TPFN of the shortest path or the minimum cost path, has been built in the picture Fuzzy setting in this article. Acting backwards from the terminal vertex, we can conveniently create the shortest path by going to the predecessor from whom the new vertex received its permanent label. Many writers have solved the shortest path problem with fuzzy arc lengths using numerous approaches. However, it appears that no research has been done on SPP using data in the form of TPFNs, a more abstract form of fuzzy numbers. This sums up the paper's main contribution. So far, there is no evidence of the suggested new technique for solving PFSPP in the literature. This motivated us to write this article and the properties used here have never been used to solve PFSPP before. This paper's key points are as follows.

This paper extends the conventional Bellman-Ford algorithm in line with the prolific researches mentioned above for calculating the minimum cost of (PFSPP) the picture fuzzy SPP. In a network with picture fuzzy arc lengths, the PFSPP aims to provide decision-makers with the length of the PFSP and the shortest path. The cost parameters are presumed to be TPFNs for transiting each arc. Based on Bellman-Ford's methods, a pseudo code for this problem is proposed. Several operating regulations and anticipated TPFN values and also score and accuracy functions are defined for comparing two TPFNs. Finally, a numerical illustration is given to explain the approach adopted and to demonstrate its capability and efficiency. Furthermore, our outcomes are explored concerning current models.

The summary of this paper is as follows. In the next section, preliminary and basic definitions are given. The purpose of the following section is to focus as an analysis of image fuzzy numbers on a few of the ideas and theories. We study their triangular and trapezoidal formulas, their methods, and the methodologies of comparison. The next section formally outlines the issue we are investigating. We introduce the Bellman-Ford algorithm and the same pseudo code. A numerical example is furnished, where our proposed $\mathrm{BA}$ is applied in the following section. In the next section, the advantages and the limitations of the proposed algorithm are given. The last section concludes the paper. 


\section{Preliminaries}

In this section, mathematical definitions that form the basis of this research are included.

\section{Definition [27]}

Let $P=\left\{\zeta,\left(m_{P}(\zeta), a_{P}(\zeta), n_{P}(\zeta)\right) \mid \zeta \in U\right\}$, in $U$ be a picture fuzzy set, where $m_{P}(\zeta) \in[0,1]$ is the positive membership degree, $a_{P}(\zeta) \in[0,1]$ is the abstinence and $n_{P}(\zeta) \in[0,1]$ is the negative membership degree of the element $\zeta$ in $U$ along with the condition $0 \leq m_{P}(\zeta)+a_{P}(\zeta)+n_{P}(\zeta) \leq 1$ and $r_{P}(\zeta)=1-\left[m_{P}(\zeta)+a_{P}(\zeta)\right.$ $\left.+n_{P}(\zeta)\right]$ is called the refusal membership value.

\section{Definition [34]}

Let $G^{*}=(P, E)$ be a graph, where $P=\left(m_{P}(\zeta), a_{P}(\zeta), n_{P}(\zeta)\right)$ is a PFS on $P$ and $E=\left(m_{E}(\zeta), a_{E}(\zeta), n_{E}(\zeta)\right)$ is a PFS on $E \subseteq V \times V$ $\exists, \forall \operatorname{arc} \zeta_{1} \zeta_{2} \in E$. The graph $G=(A, B)$ is said to be a picture fuzzy graph (PFG) on $G^{*}$ such that $m_{E}\left(\zeta_{1}, \zeta_{2}\right) \leq m_{P}\left(\zeta_{1}\right) \wedge$ $m_{P}\left(\zeta_{2}\right), a_{E}\left(\zeta_{1}, \zeta_{2}\right) \leq a_{P}\left(\zeta_{1}\right) \wedge a_{P}\left(\zeta_{2}\right), n_{E}\left(\zeta_{1}, \zeta_{2}\right) \leq n_{P}\left(\zeta_{1}\right) \vee n_{P}\left(\zeta_{2}\right)$.

\section{Definition [36]}

The ordered pair $G=(P, E)$ is said to be a picture fuzzy digraph (or) directed graph (PFDG), if $P=\left(V, m_{i}, a_{i}, n_{i}\right)$ is a PFS on $V$, where $V$ is a finite picture fuzzy non-empty set and $E=(V \times V$, $\left.m_{i, j}, a_{i, j}, n_{i, j}\right)$ is a picture fuzzy relation on $V$. Here $m_{i}: V \rightarrow[0,1]$, denotes the positive, $a_{i}: V \rightarrow[0,1]$, denotes the abstinence and $n_{i}: V \rightarrow[0,1]$, denotes the negative membership degree for every element of the vertex $v_{i}$ in $V$ along with the constraint $0 \leq m_{i}\left(v_{i}\right)+a_{i}\left(v_{i}\right)+n_{i}\left(v_{i}\right) \leq 1$. And $E \subseteq V \times V$ where $m_{i, j}$ : $V \times V \rightarrow[0,1]$ denotes the positive, $a_{i, j}: V \times V \rightarrow[0,1]$, denotes the abstinence and $n_{i, j}: V \times V \rightarrow[0,1]$, denotes the negative membership degree for every element of the edge $e_{i j}=\left(v_{i}, v_{j}\right)$ in $V$ and with the constraint $0 \leq m_{i, j}+a_{i, j}+n_{i, j} \leq 1 . m_{i, j} \leq m_{i}\left(v_{i}\right)$ $\wedge m_{j}\left(v_{j}\right), \exists a_{i, j} \leq a_{i}\left(v_{i}\right) \wedge a_{j}\left(v_{j}\right), n_{i, j} \leq n_{i}\left(v_{i}\right) \vee n_{j}\left(v_{j}\right)$.

Note: PFG has a symmetric relation on V but a PFDG do not have a symmetric relation on $V$.

\section{Definition [34]}

A picture fuzzy number $n$ is defined in a PFS, where $n \in R$, as $n=\left\{\left(\xi, m_{\mathrm{n}}(\xi), a_{n}(\xi), n_{n}(\xi)\right): \xi \in R\right\}$, where $m(\xi)=\left\{\begin{array}{l}f_{n}^{l}(\xi), \text { for } p_{1} \leq \xi<q \\ \alpha_{n}, \text { for } q \leq \xi \leq r \\ f_{n}^{u}(\xi), \text { for } r \leq \xi<s_{1} \\ 0, \text { otherwise }\end{array}\right.$

$a(\xi)=\left\{\begin{array}{l}g_{n}^{l}(\xi), \text { for } p_{2} \leq \xi<q \\ \beta_{n}, \text { for } q \leq \xi \leq r \\ g_{n}^{u}(\xi), \text { for } r \leq \xi<s_{2} \\ 0, \text { otherwise }\end{array}\right.$,

$n(\xi)=\left\{\begin{array}{l}h_{n}^{l}(\xi), \text { for } p_{3} \leq \xi<q \\ \gamma_{n}, \text { for } q \leq \xi \leq r \\ h_{n}^{u}(\xi), \text { for } r \leq \xi<s_{3} \\ 0, \text { otherwise }\end{array}\right.$.

\section{Definition [34]}

A trapezoidal picture fuzzy number (TPFN) ' $t_{n}$ ' is defined in a PFS, where $n \in R$, the set of all real numbers as $t_{\mathrm{n}}=\left\{\left(\left[p_{1}, q\right.\right.\right.$, $\left.\left.r, s_{1}\right], \alpha_{n}\right),=\left\{\left(\left[p_{2}, q, r, s_{2}\right], \beta_{\mathrm{n}}\right),=\left\{\left(\left[p_{3}, q, r, s_{3}\right], \gamma_{n}\right)\right\}\right.$, where A PFN defined in Definition 2.4 is of trapezoidal PFN if $\forall \xi$, the 3 components $\mathrm{m}$, a and $\mathrm{n}$ forms a trapezoidal shape as shown in Fig. 1. In formal terms, where

$$
\begin{aligned}
f_{n}^{l}(\xi) & =\frac{\xi-p_{1}}{q-p_{1}} \alpha_{n}^{q}, f_{n}^{u}(\xi)=\frac{s_{1}-\xi}{s_{1}-r} \alpha_{n}, \\
g_{n}^{l}(\xi) & =\frac{q-\xi+\beta_{n}\left(\xi-p_{2}\right)}{q-p_{2}}, g_{n}^{u}(\xi)=\frac{\xi-r+\beta_{n}\left(s_{2}-\xi\right)}{s_{2}-r}, \\
h_{n}^{l}(\xi) & =\frac{q-\xi+\gamma_{n}\left(\xi-p_{3}\right)}{q-p_{3}}, h_{n}^{u}(\xi)=\frac{\xi-r+\gamma_{n}\left(s_{3}-\xi\right)}{s_{3}-r} .
\end{aligned}
$$

with the constraints $0 \leq \alpha_{n}, \beta_{n}, \gamma_{n} \leq 1$ and $\alpha_{n}+\beta_{n}+\gamma_{n} \leq 1$.

\section{Definition [27]}

For the two PFNs $P_{1}=\left(m_{1}, a_{1}, n_{1}\right)$ and $P_{2}=\left(m_{2}, a_{2}, n_{2}\right)$, the operations for picture fuzzy numbers (PFNs) are given below:

1. $P_{1} \oplus P_{2}=\left(m_{1}+m_{2}-m_{1} m_{2}, a_{1} a_{2}, n_{1} n_{2}\right)$.

2. $P_{1} \otimes P_{2}=\left(m_{1} m_{2}, a_{1}+a_{2}-a_{1} a_{2}, n_{1}+n_{2}-n_{1} n_{2}\right)$.

3. $\alpha P_{1}=\left(\left(1-\left(1-m_{1}\right)\right)^{\alpha}, a_{1}^{\alpha}, n_{1}^{\alpha}\right)$.

4. $P_{1}^{\alpha}=\left(m_{1}^{\alpha}, a_{1}^{\alpha},\left(1-\left(1-n_{1}\right)\right)^{\alpha}\right)$.

\section{Definition [34]}

The score function $S(P)$ and the accuracy function $A(P)$ for a TPFN $P=([p, q, r, s], m, a, n)$ are defined as follows: 
Fig. 1 Trapezoidal picture fuzzy number

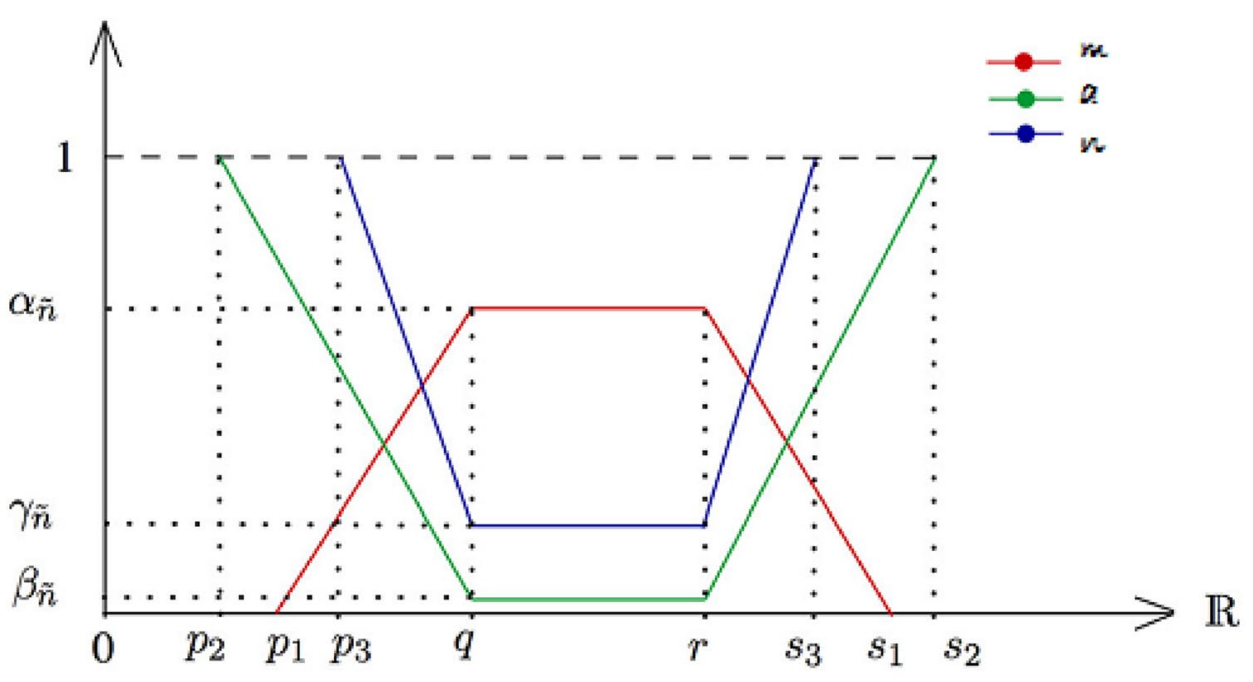

1. $E(P)=\{1 / 16[(p+q+r+s) \times(m-a-n)]\}$.

2. $S(P)=E(P) \times(m-a-n)$, where $S(P) \in[-1,1]$.

3. $A(P)=E(P) \times(m+a+n)$, where $E(P) \in[0,1]$.

Comparison of two TPFNs $P=\left(\left[p_{1}, q_{1}, r_{1}, s_{1}\right], m_{1}, a_{1}, n_{1}\right)$ and $Q=\left(\left[p_{2}, q_{2}, r_{2}, s_{2}\right], m_{2}, a_{2}, n_{2}\right)$ is done as follows:

1. if $S(P)>S(Q)$, then $P>Q$,

2. if $S(P)=S(Q)$, then,

a. if $A(P)>A(Q)$, then $P>Q$,

b. if $A(P)=A(Q)$, then $P=Q$.

\section{Network terminology}

Here, a new algorithmic method to solve PFSPP is presented.

Let us assume that $n$ number of nodes with the origin node $(\mathrm{ON}=$ node 1$)$ and end node $(\mathrm{EN}=$ node $n)$ exist. The edge set is the set that it connects any two vertices by an arrow $V=\{1,2,3, \ldots, n\}$. The picture fuzzy arc length with the nodes $\alpha$ and $\beta$ is denoted by $d \alpha \beta$ and $\operatorname{MN}(\alpha)=\{$ all nodes having the connection with the node $\alpha$ \}. For every $\alpha$ in $V-\{1\}$, it is assumed that there is at least one direction $P 1 \alpha$ in the digraph. The PFN for the edge $(\alpha, \beta)$ is defined as $d(P)=\sum d_{\alpha \beta}$ the length needed to move over $(\alpha, \beta)$ from $\alpha$ picture fuzzy distance to $\beta$ picture fuzzy distance along the line.

The SP problem's key goal is to find the least expensive path from ON " 1 " to EN " $n$ ". The unclear parameters of the SP problem under consideration are described in this work using triangular picture fuzzy numbers. As a consequence, the resulting problem is known as a picture fuzzy SP (PFSP) problem.

\section{Bellman dynamic programming (BDP): mathematical formulation}

Let us consider a picture fuzzy directed graph $G=(V, E)$ from $\mathrm{ON}$ ' 1 ' and the $\mathrm{EN}$ ' $n$ ', in which there are no cycles. The BDP system is used to determine the shortest path by the forward pass calculation process. The BDP system is defined as follows:

$g(\alpha)=\left\{\begin{array}{l}\min _{\alpha<\beta}\left[g(\alpha)+d_{\alpha \beta}\right], \text { otherwise } \\ 0, \text { if } \alpha=1\end{array}\right.$,

where $d_{\alpha \beta}$ is the directed edge $E_{\alpha \beta} s$ weight and $g(\alpha)$ is the arc length of SP from the ON 1 to the node $\alpha$. 
Algorithm 1. Picture Fuzzy Bellman-Ford algorithm Using TPFN

Picture Fuzzy Pseudo code for the BA algorithm:

1. function of Picture Fuzzy Bellman Algorithm $(G, s)$

2. $\operatorname{nrank}[\mathrm{s}] \leftarrow 0$

3. ndist $[\mathrm{s}] \leftarrow$ EmptyPFN

4. Add sinto $R$

5. For every nodei(excludings) in the PFDG G

6. Rank[i] $\leftarrow \infty$

7. Add iinto $R$

8. End For

9. $\mathrm{u} \leftarrow \mathrm{s}$

10. While( $R$ is non empty)

11. Eliminate the vertex u from $\mathrm{R}$

12. For each adjacent vertex $v$ of $u$

13. Relaxed $\leftarrow$ False

14. Temp_ndist $[v] \leftarrow$ ndist $[u]+$ edge weight of $(u, v)$

15. Temp_nrank[v] $\leftarrow \operatorname{rank}\{$ temp_ndist $[\mathrm{v}]\}$

16. Iftemp_nrank[v]<nrank[v] then

17. $n \operatorname{rank}[\mathrm{v}] \leftarrow$ temp_nrank[v]

18. Ndist[v] $\leftarrow$ temp_ndist[v]

19. $\operatorname{prev}[\mathrm{v}] \leftarrow \mathrm{u}$

20. End if

21. End for

22. If relaxed $=$ false then

23. Exit the loop

24. End if

25. $\mathrm{u} \leftarrow$ node in $\mathrm{R}$ with the minimum rankvalue

26. end while

27. for each arc $(u, v)$ PFDG G do

28. if nrank[v]>rank(ndist $[u]+$ edge weight of $(u, v)$ )

29. returnfalse

30. endif

31. endfor

32. the PFN ndist[u] is a PFN it represents the SP from the ON s to EN u

In the previous section, a numerical illustration is furnished to show the conciseness of the proposed BA technique.

In the previous section, a numerical illustration is furnished to show the conciseness of the proposed BA technique.

\section{Numerical illustration}

This section is based on an adaptable numerical problem to illustrate the possible implementation of the algorithm 
proposed.

\section{Example}

Let us consider a picture fuzzy directed network whose edge weights are expressed as a TPFN with $\mathrm{ON}=$ node 1 and $\mathrm{EN}=$ node 8 (Fig. 2). The picture fuzzy arc distance is represented in Table 1. Here we have to determine the shortest distance from $\mathrm{ON}$ to EN.

Table 2 provides the defuzzification of values of each arc weight.

Using the suggested algorithm (BA) and the Eq. (1), in Sect. 4, the SP from ON and EN is computed as follows:

$g(1)=0$.

$g(2)=\min _{\alpha<2}[g(1)+0.5175]=0+0.5175=0.5175$. $g(4)=\min _{\alpha<4}[g(3)+0.84]=-0.3225+0.84=0.5175$.

$$
\begin{aligned}
g(5) & =\min _{\alpha<5}[g(2)+0.6325, g(3)+0.492188, g(4)+0.7425] \\
= & \min [0.5175+0.6325,-0.3225+0.492188,0.5175+0.7425] \\
= & \min [1.15,0.169688,1.26]=0.169688 .
\end{aligned}
$$

$g(6)=\min _{\alpha<6}[g(5)+0.406875]=0.169688+0.406875=0.576563$.

$g(7)=\min _{\alpha<7}[g(4)+0.840938, g(5)-0.74, g(6)+0.28875]$

$=\min [0.5175+0.840938,0.169688-0.74,0.576563+0.28875]$

$=\min [1.358438,-0.570312,0.86531]=-0.570312$.

$g(8)=\min _{\alpha<8}[g(5)+0.66, g(6)+0.73125, g(7)+0.970313]$

$=\min [0.169688+0.66,0.576563+0.73125,-0.570312+0.970313]$

$=\min [0.829668,1.307813,0.400001]=0.400001$.

Fig. 2 Picture fuzzy network digraph $\mathrm{G}$

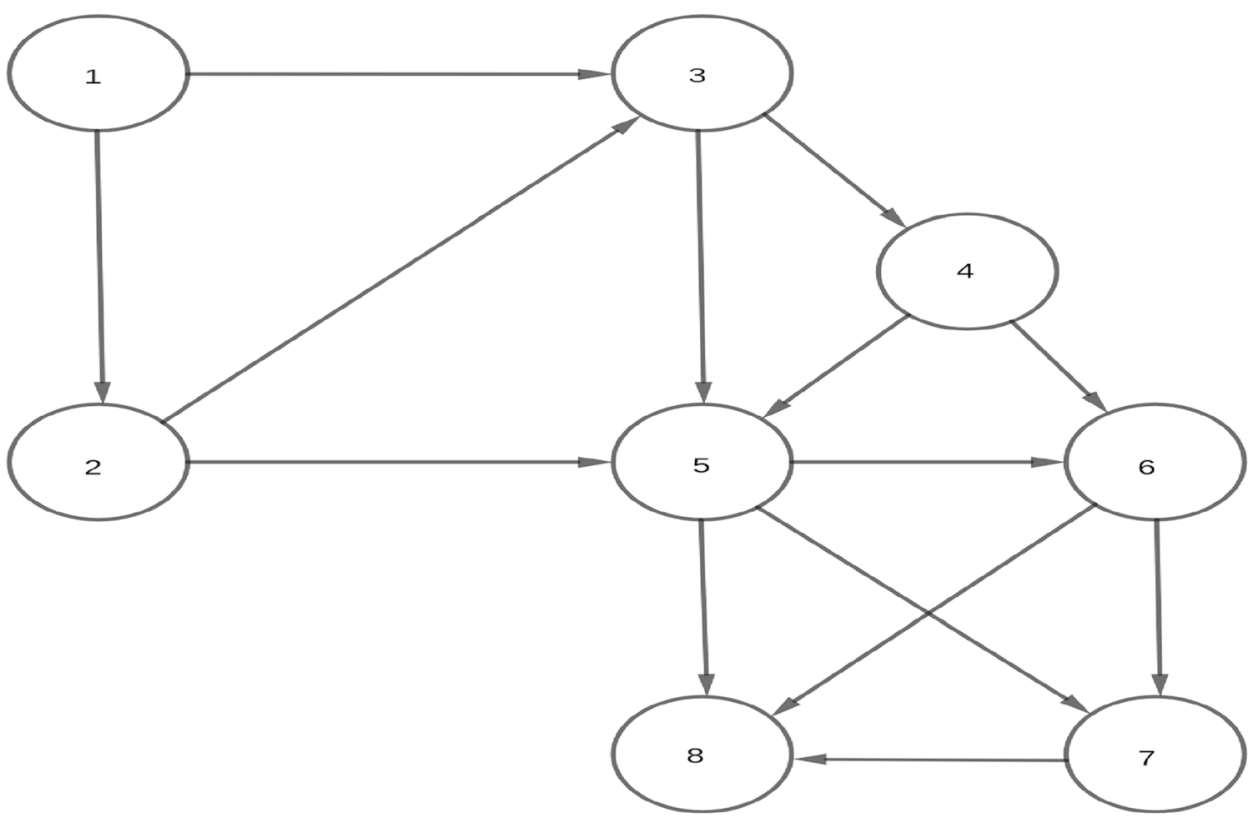

$g(3)=\min _{\alpha<3}[g(1)+0.544688$,

$g(2)-0.84]=\min [0+0.544688,0.5175-0.84]=-0.3225$.

Table 1 Arc lengths (in terms of TPFNs) of the network in Fig. 1

\begin{tabular}{llllll}
\hline S. no. & Arc name & TPFN & S. no. & Arc name & TPFN \\
\hline 1 & $(1,2)$ & $([3,5,7,9], 0.45,0.00,0.30)$ & 8 & $(4,7)$ & $([8,9,10,12], 0.50,0.10,0.20)$ \\
2 & $(1,3)$ & $([17,20,22,24], 0.40,0.25,0.10)$ & 9 & $(5,6)$ & $([12,15,17,18], 0.45,0.25,0.15)$ \\
3 & $(2,3)$ & $([7,9,12,14], 0.35,0.20,0.35)$ & 10 & $(5,7)$ & $([6,9,10,12], 0.40,0.00,0.60)$ \\
4 & $(2,5)$ & $([7,10,14,15], 0.35,0.10,0.15)$ & 11 & $(5,8)$ & $([4,5,6,7], 0.55,0.35,0.00)$ \\
5 & $(3,4)$ & $([1,2,4,5], 0.60,0.00,0.20)$ & 12 & $(6,8)$ & $([2,3,4,6], 0.60,0.10,0.20)$ \\
6 & $(3,5)$ & $([12,13,14,15], 0.55,0.30,0.15)$ & 13 & $(6,7)$ & $([7,10,13,14], 0.30,0.15,0.10)$ \\
7 & $(4,5)$ & $([20,22,24,25], 0.55,0.30,0.15)$ & 14 & $(7,8)$ & $([8,11,12,14], 0.45,0.25,0.05)$ \\
\hline
\end{tabular}


Table 2 Defuzzified arc lengths using score functions

\begin{tabular}{lllllllc}
\hline S. no. & Arc & $\mathrm{E}(\mathrm{P})$ & $\mathrm{S}(\mathrm{P})$ & $\mathrm{S}$. no. & $\operatorname{Arc}$ & $\mathrm{E}(\mathrm{P})$ & $\mathrm{S}(\mathrm{P})$ \\
\hline 1 & $(1,2)$ & 3.45 & 0.5175 & 8 & $(4,7)$ & 5.60625 & 0.840938 \\
2 & $(1,3)$ & 10.89375 & 0.544688 & 9 & $(5,6)$ & 8.1375 & 0.406875 \\
3 & $(2,3)$ & 4.2 & -0.84 & 10 & $(5,7)$ & 3.7 & -0.74 \\
4 & $(2,5)$ & 6.325 & 0.6325 & 11 & $(5,8)$ & 3.3 & 0.66 \\
5 & $(3,4)$ & 2.1 & 0.84 & 12 & $(6,8)$ & 2.4375 & 0.73125 \\
6 & $(3,5)$ & 9.84375 & 0.492188 & 13 & $(6,7)$ & 5.775 & 0.28875 \\
7 & $(4,5)$ & 7.425 & 0.7425 & 14 & $(7,8)$ & 6.46875 & 0.970313 \\
\hline
\end{tabular}

Fig. 3 Shortest path of the picture fuzzy network digraph $\mathrm{G}$

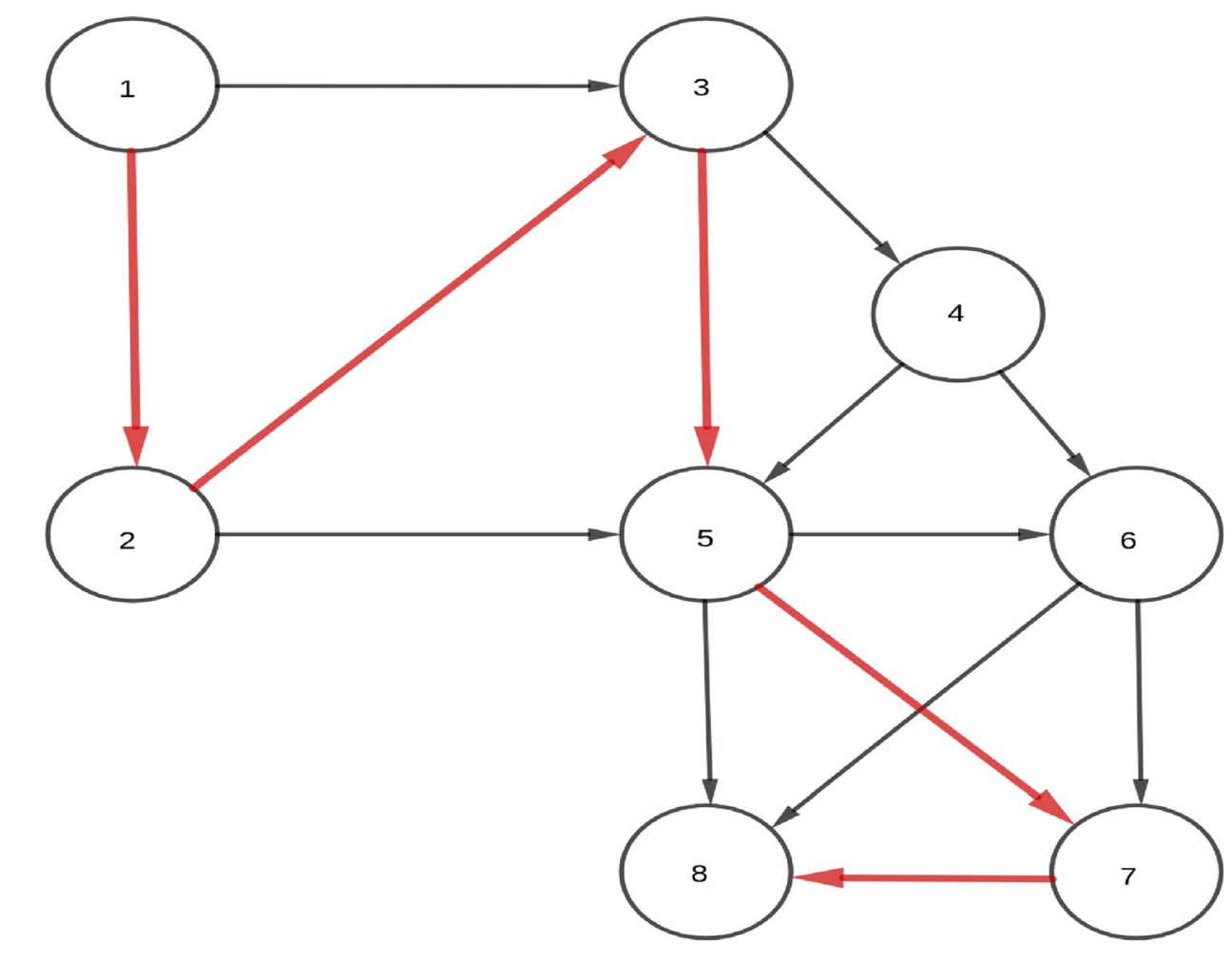

\begin{tabular}{lllll}
\hline Type of environment & Uncertainty & $\begin{array}{l}\text { Positive/truth } \\
\text { membership }\end{array}$ & $\begin{array}{l}\text { Negative/false } \\
\text { membership }\end{array}$ & $\begin{array}{l}\text { Abstain } \\
\text { member- } \\
\text { ship }\end{array}$ \\
\hline Classical graph theory & - & - & - & - \\
Fuzzy theory & $\checkmark$ & $\checkmark$ & - & - \\
Intuitionistic fuzzy theory & $\checkmark$ & $\checkmark$ & $\checkmark$ & - \\
Picture fuzzy theory & $\checkmark$ & $\checkmark$ & $\checkmark$ & $\checkmark$ \\
\hline
\end{tabular}

Table 3 Comparison analysis 
The shortest path (Fig. 3) and the shortest distance for the proposed network from the $\mathrm{ON}$ to $\mathrm{EN}$ are $1 \rightarrow 2 \rightarrow 3 \rightarrow 5 \rightarrow 7 \rightarrow 8$ and 0.400001 , respectively, via BA.

\section{Advantages and limitations of the proposed algorithm}

\section{Advantages}

- The BA operates on PFDGs with negative weight arc lengths, whereas these negative values cannot be dealt with the Dijkstra algorithm [34].

- This method can be readily put in and extended to other picture fuzzy networks with the arc weight as:

- bipolar picture fuzzy numbers;

- cubic picture fuzzy numbers;

- interval bipolar picture fuzzy numbers;

- single-valued picture fuzzy numbers;

- trapezoidal picture fuzzy numbers;

- triangular picture fuzzy numbers;

- pentagonal fuzzy numbers and so on.

\section{Limitations}

- When there is a network update, a slow response will be observed as this shift will propagate node-by-node.

- If the network contains routing loops, then there exists a node failure.

\section{Comparison table}

Table 3 shows the difference of SPP in fuzzy environment, IF environment and PF environment.

\section{Conclusion}

In this research, we have defined the PFSP where edge weights are described by TPFNs and the value of using TPFNs in PFSP is presented. The classical BA is updated by integrating the uncertainty between the origin and end nodes using TPFNs for PFSP. The main objective of this work is to define an algorithm for PFSP using TPFNS as edge weight in the picture fuzzy setting. More simply, the Bellman-Ford Algorithm has been effectively applied under the PF environment for the shortest path problem under uncertainty, and particularly, in this contribution, we have designed the SPP through the Bellman Algorithm for a network with TPFNs. We have used a basic numerical example to demonstrate the productivity of our proposed algorithm and to show that the proposed algorithm for real-life problems is very efficient, practical, and can be easily integrated with the software. The algorithms and techniques used in this study have superior advantages compared to their limitations. We believe that this study will be used in many and various other network and routing problems thanks to PF environment's power to express richer details and with the given efficient algorithm. Therefore, as future directions, the proposed algorithm can be considered for a substantially large factual SPP and our proposed algorithm to be compared with the current algorithm in terms of computational time, optimality, performance, and other aspects using software tools.

\section{Declarations}

Conflict of interest The authors declare no conflict of interest.

Open Access This article is licensed under a Creative Commons Attribution 4.0 International License, which permits use, sharing, adaptation, distribution and reproduction in any medium or format, as long as you give appropriate credit to the original author(s) and the source, provide a link to the Creative Commons licence, and indicate if changes were made. The images or other third party material in this article are included in the article's Creative Commons licence, unless indicated otherwise in a credit line to the material. If material is not included in the article's Creative Commons licence and your intended use is not permitted by statutory regulation or exceeds the permitted use, you will need to obtain permission directly from the copyright holder. To view a copy of this licence, visit http://creativecommons.org/licenses/by/4.0/.

\section{References}

1. Zadeh LA (1965) Fuzzy sets. Inf Control 8(3):338-353

2. Atanassov KT (1986) Intuitionistic fuzzy sets. Fuzzy Sets Syst 20(1):87-96

3. Zadeh LA, Information S (1975) The concept of a linguistic variable and its application to approximate reasoning I. Learn Syst Intell Robot 8(3):199-249

4. Mizumoto M, Tanaka K (1976) The four operations of arithmetic on fuzzy numbers. Syst Comput Controls 7(5):73-81

5. Dubois D, Prade H (1978) Operations on fuzzy numbers. Int J Syst Sci 9(6):613-626

6. Nahmias S (1978) Fuzzy variables. Fuzzy Sets Syst 1(2):97-110

7. Mp M, Friedman M, Kandel A (1999) A new fuzzy arithmetic. Fuzzy Sets Syst 108(1):83-90

8. Burillo P, Bustince H, Mohedano V (1994) Some definitions of intuitionistic fuzzy number. First properties. In: Proceedings of the 1st workshop on fuzzy based expert systems, Bulgaria, pp 53-55

9. Chen SM, Tan JM (1994) Handling multicriteria fuzzy decision making problems based on vague set theory. Fuzzy Sets Syst 67(2):163-172

10. Hong DH, Choi CH (2000) Multicriteria fuzzy decisionmaking problems based on vague set theory. Fuzzy Sets Syst 114(1):103-113 
11. Shu MH, Cheng CH, Chang JR (2006) Using intuitionistic fuzzy sets for fault-tree analysis on printed circuit board assembly. Microelectron Reliab 46(12):2139-2148

12. Zhang X, Liu P (2010) Method for aggregating triangular fuzzy intuitionistic fuzzy information and its application to decision making. Technol Econ Dev Econ 16(2):280-290

13. Wang JQ, Zhang Z (2008) Programming method of multi-criteria decision-making based on intuitionistic fuzzy number with incomplete certain information. Control Decis 23(10):1145-1148

14. Jianqiang W, Zhong Z (2009) Aggregation operators on intuitionistic trapezoidal fuzzy number and its application to multi-criteria decision making problems. J Syst Eng Electron 20(2):321-326

15. Maldonado Y, Castillo O, Melin P (2013) Particle swarm optimization of interval type-2 fuzzy systems for FPGA applications. Appl Soft Comput 13(1):496-508

16. Purcaru C, Precup RE, Iercan D, Fedorovici LO, David RC, Dragan F (2013) Optimal robot path planning using gravitational search algorithm. Int J Artif Intell 10(S13):1-20

17. Bellman R (1958) On a routing problem. Q Appl Math 16(1):87-90

18. Dijkstra EW (1959) A note on two problems in connexion with graphs. Numer Math 1(1):269-271

19. Warshall S (1962) A theorem on Boolean matrices. J ACM 9:11-12

20. Floyd RW (1962) Algorithm 97: shortest path. Commun ACM 5(6):345

21. De PK, Bhincher A (2011) Dynamic programming and multi objective linear programming approaches. Appl Math Inf Sci 5(2):253-263

22. Kumar G, Bajaj RK, Gandotra N (2015) Algorithm for shortest path problem in a network with interval-valued intuitionistic trapezoidal fuzzy number. Proc Comput Sci 70:123-129

23. Meenakshi AR, Kaliraja M (2012) Determination of the shortest path in interval valued fuzzy networks. Int J Math Arch 3(6):2377-2384

24. Elizabethand S, Sujatha L (2014) Fuzzy shortest path problem based on interval valued Fuzzy number matrices. Int J Math Sci Eng Appl 8(1):325-335
25. Das D, De PK (2014) Shortest path problem under intuitionistic fuzzy setting. Int J Comput Appl 105(1):1-4

26. Alcantud JCR, Laruelle A (2014) Dis \& approval voting: a characterization. Soc Choice Welf 43(1):1-10

27. Cuong BC (2013) Picture fuzzy sets-first results, part 1. In: Seminar neuro-fuzzy systems with applications. Preprint 03/2013. Institute of Mathematics, Vietnam Academy of Science and Technology, Hanoi

28. Cuong BC (2013) Picture fuzzy sets-first results. Part 2, seminar neuro-fuzzy systems with applications. Institute of Mathematics, Hanoi

29. Cuong BC, Kreinovich V (2013) Picture fuzzy sets-a new concept for computational intelligence problems. In: 2013 third world congress on information and communication technologies (WICT 2013). IEEE, pp 1-6

30. Singh P (2015) Correlation coefficients for picture fuzzy sets. J Intell Fuzzy Syst 28(2):591-604

31. Thong PH (2017) Some novel hybrid forecast methods based on picture fuzzy clustering for weather nowcasting from satellite image sequences. Appl Intell 46(1):1-15

32. Thong PH (2015) A new approach to multi-variable fuzzy forecasting using picture fuzzy clustering and picture fuzzy rule interpolation method. In: Knowledge and systems engineering. Springer, Cham, pp 679-690

33. Akram M, Zafar F (2020) Hybrid soft computing models applied to graph theory. Stud Fuzz Soft Comput 380:1-434

34. Akram M, Habib A, Alcantud JCR (2020) An optimization study based on Dijkstra algorithm for a network with trapezoidal picture fuzzy numbers. Neural Computing and Applications (Springer) 33:1329-1342

35. Mani P, Broumi S, Muthusamy K (2019) A network shortest path algorithm via hesitancy fuzzy digraph. J New Theory 27:52-62

36. Mani P, Vasudevan B, Sivaraman M (2021) Shortest path algorithm of a network via picture fuzzy digraphs and its application. Materials Today: Proceedings 45:3014-3018

Publisher's Note Springer Nature remains neutral with regard tojurisdictional claims in published maps and institutional affiliations. 